\title{
Fourth Replication of Saffran, Newport, \& Aslin (1996) Word segmentation: The role of distributional cues, Exp. 1
}

\author{
Han Xiang P. Choong ${ }^{1}$, Tiwalayo Eisape ${ }^{1}$, Shelby A. Grasso ${ }^{1}$, Lisa C. Kurt ${ }^{1}$, Celine Jia Rong Lim ${ }^{1}$, \\ Jingrun Lin ${ }^{1}$, Carrie E. Milinazzo ${ }^{1}$, Yueran Yang ${ }^{1}$, Mariela Jennings ${ }^{1}$, Lauren Skorb ${ }^{1}$, \& Joshua K. \\ Hartshorne $^{1}$ \\ ${ }^{1}$ Boston College
}

\begin{abstract}
These are the results for an online replication of Saffran, Newport, \& Aslin (1996) Word segmentation: The role of distributional cues, Journal of Memory and Language, 35, 606-621. This replication follows two online replications and an in-lab replication of the same experiment (Hartshorne 2017, Replication of Saffran, Newport, \& Aslin (1996) Word segmentation: The role of distributional cues, Exp. 1. PsyArXiv doi:10.17605/OSF.IO/E5C64).
\end{abstract}

Keywords: statistical learning, unsupervised learning, language acquisition, replication

\section{Introduction}

In this paper, we describe the methods and results for an online replication of Experiment 1 from a classic statistical word-segmentation paper by Saffran, Newport, and Aslin (1996) (henceforth: SNA96). This experiment is part of a larger project to systematically replicate as many experiments involving statistical word learning in adults as possible and follows two online replications of the same study (Garcia et al., 2017; Hartshorne, 2017) and one in-lab replication. The present study uses the materials from the second online replication (Hartshorne, 2017) and the in-lab replication.

\section{Method}

\section{Subjects}

All subjects were native speakers of English and were recruited through Amazon Mechanical Turk for monetary compensation. We ran two sets of analyses, one set which included all recruited participants and another set which excluded participants who missed any catch trials. We included the catch trials as an attention screen in order to confirm that the results were not due to subjects who were not paying attention. In our previous replication, the results from our analyses with all subjects did not differ from the results from our analyses with only attentive subjects (Hartshorne, 2017).

All subjects. We ran a total of 172 subjects; 72 subjects in the partword condition (Ages 23-69, M=38.82) and 100 in

Correspondence concerning this article should be addressed to Joshua K. Hartshorne, McGuinn Hall. E-mail: info@13atbc.org the nonword condition (Ages 22-64, M=36.15).

Attentive subjects. For the set of analyses with the applied attention screen, we excluded the 69 subjects ( 50 or $50 \%$ in the nonword condition and 19 or $26.39 \%$ in the partword condition) who missed any catch trials. Note that the catch trials were relatively subtle, so this is a strong filter.

After exclusions, we included a total of 103 subjects; 53 subjects in the partword condition (Ages 23-69, M=40.72) and 50 in the nonword condition (Ages 23-64, M=38.47).

\section{Known differences with the original.}

- Our study had an order of magnitude more subjects (172 vs. 24) and thus considerably more power and precision.

- Subjects in our study were significantly older on average than the original subjects, who were all undergraduates.

- Subjects in our study were compensated monetarily rather than with course credit.

\section{Materials}

We used the same materials from our previous online and in-lab replications (Hartshorne, 2017).

The language constructed for the experiment consisted of four consonants $(/ \mathrm{p} /, / \mathrm{t} /, / \mathrm{b} /, / \mathrm{d} /)$ and three vowels $(/ \mathrm{a} /, / \mathrm{i} /$, $/ \mathrm{u} /$ ) which, when combined, rendered an inventory of $12 \mathrm{CV}$ syllables. We combined eleven of these syllables (excluding /di/) to create six trisyllabic words: babupu, bupada, dutaba, patubi, pidabu, and tutibu. Some members of the syllable inventory occurred in more words than others; for example, /bu/ occurred in four words, while/ta/ occurred in only one. 
This ensured varying transitional probabilities within the words themselves.

We generated each syllable using MBROLA (Dutoit, Pagel, Pierret, Bataille, \& Van der Vrecken, 1996) with voice us 1 and with full coarticulation between syllables. For each syllable, the consonant lasted for $100 \mathrm{~ms}$ and the vowel for $177 \mathrm{~ms}$, for a rate of 216 syllables/minute. We concatenated 252 tokens of each of the 6 words in a random order, with the stipulation that the same word never occurred twice in a row. We converted the resulting sound file from WAV to MP3 using SoX (Bagwell \& Contributors, 2015).

Average transitional probability within a word ranged from $43.0 \%$ to $100 \%$. Transitional probabilities between phonemes spanning words ranged from $5.0 \%$ to $59.0 \%$.

For the test phase, we created 6 nonword foils and 6 partword foils. The nonwords (pibuda, badapu, tapubi, bubita, dubiti, bubibi) consisted of syllables from the language's syllable inventory which never followed each other in the speech stream, even across word boundaries. Thus, the transitional probabilities between each of the syllables in the nonwords were zero.

Each of the 6 partwords consisted of a syllable pair from a word from the language, plus an additional syllable. For example, the final syllable of the word pidabu was altered to create the partword pidata. Similarly, the first syllable of the word dutaba was altered to create the partword bitaba. Three partwords contained the first two syllables of words (pidata, bupabi, babuda) and three contained the final two syllables of words (bitaba, datubi, titibu).

We generated the stimuli for the test phase from the same syllables used to create the training, using the procedure described above. We created 72 test trials by combining each real word with each foil, with an approximately 500 ms gap in between, with the stipulation that the real word appeared first in half of the trials in each condition (nonword or partword).

In addition, we generated 6 catch trials, which pitted each word against a (different) foil that contained multiple phonemes that did not appear in the training.

\section{Known differences with the original.}

- The original stimuli were produced with MacinTalk. We used MBROLA.

- We used all three of the partwords that are explicitly mentioned in the original paper (pidata, bitaba, bupabi). Otherwise, our foils likely differ from theirs.

- The original paper states that training included 300 tokens of each of the 6 words at 216 syllables/minute for a total of 21 minutes. These numbers are mutually incompatible: Either the speed was faster (257 syllables/minute), the length of the audio was longer (25 $\min$ ), or there were fewer tokens (252). The latter number matches the assertion elsewhere in the same paragraph that there were a total of 4,536 syllables. In this way, we presented 252 tokens of each of the 6 words in order to have 21 minutes at 216 syllables/minute.

- The original paper reports that the transitional probabilities between phonemes spanning words ranges from $10 \%$ to $20 \%$. This is inconsistent with the rest of the reported method. If they constructed training as described, the transitional probability between the words dutaba and bupada would have to be at least $50 \%$. This follows from that fact that in at least $50 \%$ of the cases where /ba/ appears, it is within the word babupu. Hence, if bupada ever followed dutaba in training, the transitional probability of / babu/ would be greater than $50 \%$. The probability of that not having happened is negligible $(p<4 \mathrm{e}-25)$. For similar reasons, the transitional probability between the words pidabu and patubi would be around $35 \%$. The transitional probabilities between phonemes spanning words in our experiment ranged from $5.0 \%$ to $59.0 \%$.

- The average transitional probability within-word were $42.0 \%, 42.5 \%, 50.0 \%, 75.0 \%, 75.0 \%$, and $100 \%$. The original paper reports $37 \%, 41 \%, 50 \%, 75 \%, 75 \%$, and $100 \%$. The difference could be due to differences in the random orders of the stimuli or due to a miscalculation on the part of the original authors.

\section{Possible differences with the original.}

- In our version, all six of the real words were used to create partwords. The original paper does not specify whether all words were used to create partwords.

- In our version, we balanced the number of test trials where the correct answer came first or second. No information regarding this issue is provided in the original paper.

\section{Apparatus}

We programmed the experiment using jsPsych (Leeuw, 2014). As the experiment was conducted online, we were unable to control the listening environment of the participants on Mechanical Turk, but instructed them before the experiment to sit in a quiet and non-distracting environment with the sound turned up to a sufficient level.

\section{Known differences with the original.}

- The original experiment was conducted in an IAC sound-attenuated booth. Stimuli were presented using an Aiwa tape deck and a Proton speaker. The test phase 
was administered on a Macintosh IIsi computer, using the PsychLab stimulus presentation package.

\section{Procedure}

Participants were instructed to listen to a "nonsense" language and were told that the language contained words, but no meanings or grammar. They were informed that their task was to figure out where the words began and ended. Subjects were given no information about the length or structure of the words or how many words the language contained. They were informed that the listening phase of the experiment consisted of three short blocks, followed by a test of their knowledge of the words in the language. Subjects were allowed to take a break after each of the first two 7-min listening blocks.

After a total of $21 \mathrm{~min}$ of listening, subjects received a two-alternative forced-choice test. For each test item, subjects heard two trisyllabic strings, separated by $500 \mathrm{~ms}$ of silence. One of these strings was a word from the nonsense language, while the other was not. Subjects were asked to indicate which of the two strings sounded more like a word from the language by pressing either the " 1 " or " 2 " key on the keyboard. The test items were constructed by pairing the six words of the language with one of two types of trisyllabic foils. For half of the subjects, the six nonword foils were illegal strings of syllables from the language. For the other half of the subjects, the six foils were partwords, consisting of a syllable pair from a word plus an additional syllable. After the subject's response, the next item was presented. We randomized the order of the presentation of the items.

After the primary test trials, all subjects answered the 6 catch trials. The order was randomized for each subject. The catch trials followed the primary test trials immediately, without any overt distinction.

\section{Known differenes with the original}

- The original study enforced a five-minute break between training blocks. Given the nature of online testing, this was difficult to enforce, so we allowed subjects to self-pace their breaks.

- The original provided practice trials were given to each subject prior to the test, in order to clarify the structure of the test and to give subjects practice with the key presses. The practice trials consisted of strings of syllables which were not part of the language's syllable inventory. We did not include practice trials, as it seemed unlikely that participants in an online study were unfamiliar with computers.

- The original study did not include catch trials. Note that because the catch trials follow the primary test trials,
Test Phase Accuracy by Condition (All Subjects)

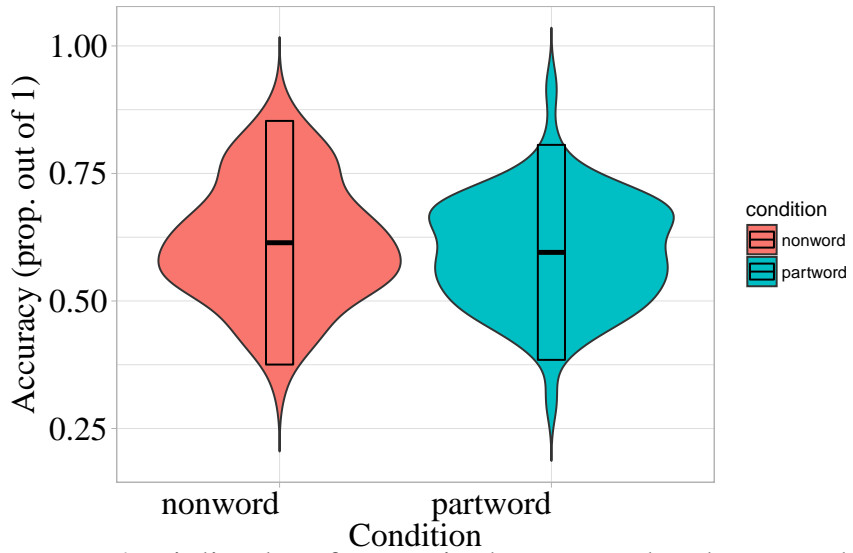

Figure 1. Violin plot of scores in the nonword and partword conditions in the present study. Note that all subjects are included.

they cannot affect the results of those trials (except in that we can use the catch trials to distinguish poor learners from noncompliant subjects).

\section{Results}

\section{All subjects}

Original Analyses. SNA96 report that the mean score in the nonword test condition was 27.2 out of 36 (76\%), and a single sample $t$-test found that performance was significantly above chance $(t(11)=6.78, p<.01)$. They also report that all six words were learned better than chance ( $p<.01$ for each word). For the partword test condition, SNA96 report that the mean score was 22.3 out of $36(65 \%)$, which was significantly above chance $(t(11)=3.38, p<$ $.01)$. They report that three of the six words were learned significantly better than chance $(p<.01$ for two words and $p<.05$ for one word). SNA96 found that partword performance was significantly lower than performance in the nonword test condition $(t(22)=2.66, p<.05)$.

The overall results from the present replication for all subjects (before applying any exclusion criteria) are presented in Figure 1. In the nonword test condition, the mean score was 22.11 of a possible $36(0.61, \mathrm{SE}=0.01)$, where chance performance equals 18. A single-sample $t$-test (two-tailed) with equal variances showed overall performance significantly different from chance $(t(99)=9.56, p=1 \mathrm{e}-15)$. 5 of the 6 words (bupada, dutaba, patubi, pidabu, tutibu) in this condition were learned at a level significantly better than would be expected by chance ( $p<0.05$ for each word).

Although the foils in the partword condition were designed to be more confusable with the words, the mean score for 
participants was 21.43 of a possible $36(0.60, \mathrm{SE}=0.01)$, a rate that significantly exceeded chance $(t(71)=7.67$, $p=6.7 \mathrm{e}-11$ ). 5 of the 6 words (babupu, dutaba, patubi, pidabu, tutibu) were learned significantly better than would be expected by chance $(p s<.01)$. Success did not significantly differ from that in the nonword condition $(t(170)=1.07, p=0.28)$. We replicate the finding of statistical word segmentation, but we do not replicate the effect of test condition (nonword vs. partword).

Next, we asked whether the strengths of the transitional probabilities played a role in which words were learned better than others. Recall that the transitional probabilities between the pairs of syllables within words ranged between .43 and 1. We split the six words into two sets, one set containing the three words with the highest average transitional probabilities (1.0, .77, and .75), and the other set containing the three words with the lowest average transitional probabilities $(.50, .43$, and .43$)$. SNA96 report that subjects performed significantly better on the three words with the high transitional probability (79\%) than on the three words with the low transitional probability $(72 \%)$, a difference that reached significance $(t(11)=2.31, p<.05)$.

In the present replication, a matched-pairs $t$-test with equal variances comparing subjects' performance on the three words with the highest transitional probabilities ( $M=0.62$, $\mathrm{SE}=0.01)$ to the three words with the lowest transitional probabilities $(M=0.60, S E=0.01)$ indicated no significant difference in performance: $t(171)=1.29, p=0.2$. We do not replicate the original finding that words with higher transitional probabilities were more easily recognized.

SNA96 report that accuracy was particularly high on partword trials with the foil bupabi (false-alarm rate of $26 \%$ ). This is relevant because during training, the syllable pair /bupa/ was more common than any other. If subjects were simply following bigram frequencies, they should have relatively poor performance on this item. Similar to SNA96, we found that the false alarm rate on such trials was lower than chance (mean false alarm rate of $0.39, \mathrm{SE}=0.03$; $t(71)=4.12, p=1 \mathrm{e}-04)$. We replicate this original finding of low false-alarm rates with the foil bupabi, although we find a higher false alarm rate than SNA96.

These results may be probed further to determine which aspects of the words were learned. Recall that the partword test used two types of partwords. Half of the partwords contained the first two syllables of words plus a final syllable, and the other half contained the final two syllables of words plus an initial syllable. SNA96 found that while the first type produced false alarm rates significantly lower than chance $(\mathrm{M}=29 \% ; t(11)=-61.3, p<.01)$, the latter did not $(\mathrm{M}=48 \% ; t(11)=-.39, p>.05)$, a difference that reached significance $(t(11)=-3.25, p<.01)$.

We find below-chance false alarm rates for change-final foils $(\mathrm{M}=0.39, t(71)=-7.16, p=6 \mathrm{e}-10)$ and changefirst foils $(\mathrm{M}=0.42, t(71)=-5.35, p=1 \mathrm{e}-06)$, with a difference that just reaches significance $(t(71)=2.00, p$ $=0.05$ ). We replicate the significant difference in false alarm rates in the change-first versus change-final foils, although we find below-chance false alarm rates for both change-final and change-first foils, while SNA96 found below-chance performance for only change-final foils.

Revised Analyses. There are two important limitations to the original analyses conducted by SNA96. First, their use of $t$-tests on accuracy data violates core assumptions of $t$-tests: that the data are continuous and error is normally distributed. Violations of these assumptions can lead both to false positives and false negatives (Jaeger, 2008). Binomial models are more appropriate (Jaeger, 2008). ${ }^{1}$

Additionally, they treat items as fixed effects. Therefore, any conclusions are specific to the specific stimuli used. No assessment of generalization can be made. Statisticians have suggested two options. The first is to conduct analyses separately over subject averages (as SNA96 did) and over item averages (Clark, 1973). The second option is to use mixed effects models (Baayen, Davidson, \& Bates, 2008).

Given these considerations, we re-analyzed the data using binomial mixed effects models, implemented in $\mathrm{R}$ using the lme4 package, ver. 1.1-12 (D. Bates et al., 2017). Our first model had a single fixed effect: foil type (nonword/partword). We considered two possible random effects structures. The first estimated separate random intercepts for subject and each word and foil separately, plus a random slope of foil type for each word. The second estimated random intercepts for subject and each word/foil pair. The model with the second random effects structure had a lower Akaike's Information Criterion (AIC) than the model with the first random effects structure (Change in AIC $=10$ ), so we use the second random effects structure in these analyses.

We assessed the effect of foil type with a binomial mixed effects model with a fixed effect of foil type and random effects as described above. The main effect of foil type was not significant (Wald's $z=-0.08$, $p=0.44)^{2} \quad$ These results match our results from

\footnotetext{
${ }^{1} \mathrm{An}$ intermediate option is to transform accuracy measures to log-odds, though this method is less accurate than a binomial model.

${ }^{2}$ Wald's $z$ is somewhat anti-conservative when sample size is small [@luke2016evaluating]. Thus, these analyses may, if anything,
} 
the original analyses; we do not replicate SNA96's reported effect of condition (nonword vs. partword).

We further assessed evidence for learning by running the above model with no fixed effects. The intercept was significant when collapsing across conditions (Wald's $z=$ $0.45, p=5.5 \mathrm{e}-17)$, or when considering the nonword and partword condition separately (respectively: Wald's $z=0.49$, $p=8.4 \mathrm{e}-12$; Wald's $z=0.40, p=3.4 \mathrm{e}-07$ ), indicating that accuracy was significantly different from chance. These results match the results from the original analyses and replicate the original finding of statistical word segmentation.

Next, we asked whether the three words with higher transitional probability were recognized at a different rate from the three with lower transitional probability. For this model, we included a fixed effect of transitional probability and random effects as described above. The main effect of transitional probability (high vs. low) was not significant (Wald's $z$ $=-0.07, p=0.45$ ). These results match the original analyses; we do not replicate this finding from SNA96.

We also ran the model with no fixed effects, looking only at performance with the foil bupabi. We find that performance on these trials was significantly above chance (Wald's $z=$ $0.47, p=4.2 \mathrm{e}-04$ ), and we therefore replicate the original effect of high accuracy on trials with the foil bupabi, which aligns with our results from the original analyses.

Finally, we compared false alarms to the partwords that matched real words on the first two syllables against accuracy for those that matched real words on the last two syllables with a model with a fixed effect for partword type and random effects as described above. The main effect of partword type was not significant (Wald's $z=-0.15, p=0.29$ ). These results do not match the results from the original analyses: Unlike the results from the original analyses above, we fail to replicate the original finding.

\section{Excluding in-attentive subjects}

Original Analyses. The overall results from the present replication after excluding all participants who missed any catch trials are presented in Figure 2. In the nonword test condition, the mean score was 23.70 of a possible 36 (0.66, SE=0.02), where chance performance equals 18. A single-sample $t$-test (two-tailed) with equal variances showed performance significantly different from chance $(t(49)=9.38, p=1.6 \mathrm{e}-12) . \quad 5$ of the 6 words (bupada, dutaba, patubi, pidabu, tutibu) in this condition were learned at a level significantly better than would be expected by chance $(p<0.05$ for each word).

Although the foils in the partword condition were designed
Test Phase Accuracy by Condition (Only Attentive Subjects)

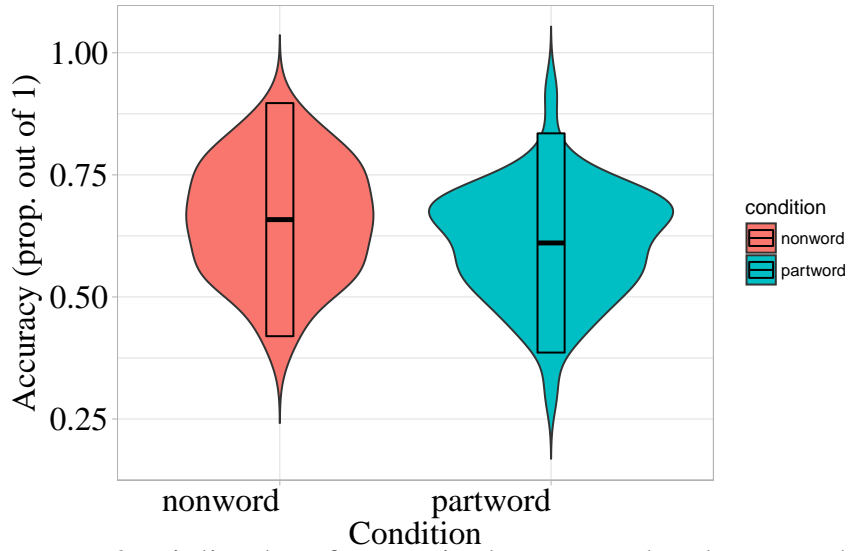

Figure 2. Violin plot of scores in the nonword and partword conditions in the present study. Note that only attentive subjects are included.

to be more confusable with the words, the mean score for participants was 21.98 of a possible $36(0.61, \mathrm{SE}=0.02)$, a rate that significantly exceeded chance $(t(52)=7.17, p=$ 2.6e-09). 4 of the 6 words (babupu, patubi, pidabu, tutibu) were learned significantly better than would be expected by chance $(p s<.05)$. Success did not significantly differ from that in the nonword condition $(t(101)=2.09, p=$ 0.3 ). These results match the results (both original and revised) from all subjects above; we replicate the effect of statistical word segmentation, and we do not replicate the original effect of condition (nonword vs. partword).

Next, we asked whether the strengths of the transitional probabilities played a role in which words were learned better than others. Recall that the transitional probabilities between the pairs of syllables within words ranged between .43 and 1 . We split the six words into two sets, one set containing the three words with the highest average transitional probabilities $(1.0, .77$, and .75), and the other set containing the three words with the lowest average transitional probabilities $(.50, .43$, and .43). A matched-pairs $t$-test with equal variances comparing subjects' mean performance on the three words with the highest transitional probabilities $(\mathrm{M}=0.65$, $\mathrm{SE}=0.02)$ to the three words with the lowest transitional probabilities $(\mathrm{M}=0.61, \mathrm{SE}=0.01)$ indicated no significant difference in performance on the words containing higher transitional probabilities: $t(102)=1.97, p=0.051$. We do not replicate the original finding that words with higher transitional probabilities were more easily recognized, and these results align with our previous results from all subjects above (both original and revised analyses).

Similar to SNA96, we found that performance on trials overstate the evidence against the null. 
with the foil bupabi was higher than chance ( $\mathrm{M}=0.61$, $\mathrm{SE}=0.03 ; t(52)=3.50, p=9.5 \mathrm{e}-04)$. We replicate this original finding, which aligns with the results from all subjects (both original and revised analyses).

We find below-chance performance for both change-final foils $(\mathrm{M}=0.37, t(52)=-6.51, p=3 \mathrm{e}-08)$ and change-first foils $(\mathrm{M}=0.41, t(52)=-5.14, p=4.2 \mathrm{e}-06)$, with no significant difference between the two $(t(52)=1.88, p=0.07)$. We do not replicate the original finding, which align with the revised analyses from all subjects, but differs from the results using the original analyses with all subjects.

Revised Analyses. Given the considerations described above, we re-analyzed the data using binomial mixed effects models, implemented in $\mathrm{R}$ using the lme4 package, ver. 1.1-12 (D. Bates et al., 2017). Our first model had a single fixed effect: foil type (nonword/partword). We considered two possible random effects structures. The first estimated separate random intercepts for subject and each word and foil separately, plus a random slope of foil type for each word. The second estimated random intercepts for subject and each word/foil pair. The model with the first random effects structure had a lower Akaike's Information Criterion (AIC) than the model with the second random effects structure (Change in AIC =9), so we use the second model and its random effects structure throughout these analyses.

We assessed the effect of foil type with a binomial mixed effects model with a fixed effect of foil type and random effects as described above. The main effect of foil type was not significant (Wald's $z=-0.23, p=0.29$ ). These results match all previous analyses; we do not replicate the original effect of condition (partword vs. nonword).

We further assessed evidence for learning by running the above model with no fixed effects. The intercept was significant when collapsing across conditions (Wald's $z=$ $0.59, p=1.5 \mathrm{e}-04$ ), or when considering the nonword and partword condition separately (respectively: Wald's $z=0.71$, $p=6.4 \mathrm{e}-04$; Wald's $z=0.48, p=5.2 \mathrm{e}-03$ ), indicating that accuracy was significantly different from chance. These results match all previous analyses (with and without exclusions using both the original and revised analyses); we replicate the effect of statistical word segmentation.

Next, we asked whether the three words with higher transitional probability were recognized at a different rate from the three with lower transitional probability. For this model, we included a fixed effect of transitional probability and random effects as described above. The main effect of transitional probability (high vs. low) was not significant (Wald's $z=$
$-0.20, p=0.44)$. Similar to our results from all previous analyses, we fail to replicate the original results that words with high transitional probabilities are learned better than words with low transitional probabilities.

We also ran the model with no fixed effects, looking only at performance with the foil bupabi. We use the random effects structure described above, except we do not include the random intercept of foil because these analyses included only one foil type. We find that performance on these trials was significantly above chance (Wald's $z=0.50, p=3.8 \mathrm{e}-03$ ), and we therefore replicate the original effect of high accuracy on trials with the foil bupabi, which aligns with our results from all previous analyses (original and revised analyses using all subjects and only attentive subjects).

Finally, we compared false alarms to the partwords that matched real words on the first two syllables against accuracy for those that matched real words on the last two syllables with a model with a fixed effect for partword type and random effects as described above. The main effect of partword type was not significant (Wald's $z=-0.19, p=$ 0.41 ). Similar to our results from the original analyses and the revised analyses with all subjects, we fail to replicate the original results that false alarm rates are lower with change-final foils than with change-first foils.

\section{Discussion}

To summarize, we replicate the finding of statistical word segmentation (both separately by condition and collapsed across condition), and we find this effect when we use both the original and revised analyses and when considering all subjects and only attentive subjects.

SNA96 report several moderating effects, as well: They report that accuracy in the partword condition is lower than accuracy in the nonword condition, words with high transitional probabilities are learned better than words with low transitional probabilities, the foil bupabi, which includes the most common bigram /bupa/, produced below-chance false-alarm rates, and partwords that differed from real words by only the last syllable produced fewer false-alarms than partwords that differed from real words by only the first syllable.

Of all of these reported moderating effects, the only effect that we find consistent evidence for is that performance on trials with the foil bupabi was greater than chance. We replicate this finding using both the original and revised analyses and when considering both all subjects and only attentive subjects. We also replicate the original finding that change-final partwords produce fewer false alarms than change-first partwords, but we are only able to replicate this finding using the original 
analyses on all subjects. We fail to replicate all remaining moderating effects across all analyses.

\section{References}

Baayen, R. H., Davidson, D. J., \& Bates, D. M. (2008). Mixedeffects modeling with crossed random effects for subjects and items. Journal of Memory and Language, 59(4), 390-412.

Bagwell, C., \& Contributors, S. (2015). SoX: Sound eXchange, the swiss army knife of audio manipulation. sox.sourceforge.net.

Bates, D., Maeschler, M., Bolker, B., Walker, S., Christensen, R. H. B., Singmann, H., ... Green, P. (2017). Linear mixed-effects models using 'eigen' and s4. cran.rproject.org/web/packages/lme4/index.html.

Clark, H. H. (1973). The language-as-fixed-effect fallacy: A critique of language statistics in psychological research. Journal of Verbal Learning and Verbal Behavior, 12(4), 335359.

Dutoit, T., Pagel, V., Pierret, N., Bataille, F., \& Van der Vrecken, O. (1996). The mbrola project: Towards a set of high quality speech synthesizers free of use for non commer- cial purposes. In Proceedings of the Fourth International Conference on Spoken Language (Vol. 3, pp. 1393-1396). IEEE.

Garcia, C. R., Iozzo, G. L., Lamirato, K. E., Ledoux, J. R., Mu, J., Murdock, K. N., ... Hartshorne, J. K. (2017). Replication of Saffran, Newport, \& Aslin (1996) Word segmentation: The role of distributional cues, exp. 1. doi:10.17605/OSF.IO/QSYD2

Hartshorne, J. K. (2017). Replication of saffran, newport, \& aslin (1996) word segmentation: The role of distributional cues, exp. 1. doi:10.17605/OSF.IO/E5C64

Jaeger, T. F. (2008). Categorical data analysis: Away from ANOVAs (transformation or not) and towards logit mixed models. Journal of Memory and Language, 59(4), 434-446. doi:10.1016/j.jml.2007.11.007

Leeuw, J. R. de. (2014). JsPsych: A JavaScript library for creating behavioral experiments in a web browser. Behavior Research Methods, 47(1), 1-12.

Saffran, J. R., Newport, E. L., \& Aslin, R. N. (1996). Word segmentation: The role of distributional cues. Journal of Memory and Language, 35(4), 606-621. 